\title{
Chemorepulsion and thymocyte emigration
}

\author{
Jason G. Cyster

\begin{abstract}
Howard Hughes Medical Institute, and Department of Microbiology and Immunology, University of California, San Francisco, 513 Parnassus Ave., San Francisco, California 94143-0414, USA. Phone: (415) 502-6427; Fax: (415) 502-8424; E-mail: cyster@itsa.ucsf.edu.
\end{abstract}

Commentary

See related article, pages $1101-1110$.

J. Clin. Invest. 109:1011-1012 (2002). DOI:10.1172/JCI200215511.

The possibility that chemorepulsion, or cell migration away from a stimulus, plays a role in the immune system is exciting from both clinical and cellbiological viewpoints. For the clinician, what could be a more satisfying way to treat a cell-mediated immune disease than local application of a chemorepellent? For the cell biologist, eukaryotic cell chemorepulsion represents a novel behavior to be explored and explained at the molecular level. Although the repulsion of neurons and their axons has emerged as an important process in patterning the nervous system (1), a physiological role for chemorepulsion in directing movement in the immune system has yet to be revealed. Therefore, any evidence that chemorepulsion regulates immunocyte migration demands close attention.

In this issue of the JCI, Poznansky and coworkers suggest that chemorepulsion, which they term "fugetaxis," plays a role in T cell emigration from the thymus (2). This group first reported evidence for immune cell chemorepulsion in a study of human peripheral blood $\mathrm{T}$ cells. In both Transwell chemotaxis chamber assays and a methylcellulose migration assay, they

\section{Poznansky and coworkers suggest that chemorepulsion, \\ which they term "fugetaxis," plays a role \\ in T cell emigration from the thymus.}

showed that $\mathrm{T}$ cells could migrate away from a high concentration source of the chemokine SDF1/CXCL12 (3). Experiments in the Transwell system, where cells were incubated in the same high concentrations of SDF1/CXCL12 in the absence of a gradient, provided evidence that the migration was not simply due to chemokinesis, in which cellular motility increases without any directional specificity.

Like SDF1/CXCL12-mediated chemotaxis, the putative chemorepulsion/fugetaxis behavior is sensitive to pertussis toxin (PTX), implicating Gimediated signaling in this process. These observations suggested not only that a cell can sense differences in chemokine receptor occupancy between its front and back, but also that it can respond differently depending on the extent of receptor occupancy. Indeed, Zlatopolskiy and Laurence (4), noting that the SDF1/CXCL12 receptor CXCR4 binds its ligand with high and low affinity sites, have proposed that binding to the lower affinity site is required to induce a chemorepulsive signal. Such a mechanism would distinguish this process from the bestcharacterized examples of chemorepulsion, found in the nervous system (1). There, the type of response is determined by the type of receptor expressed by the cell, rather than by ligand concentration (5). It is important to recall, however, that only a fraction of the $\mathrm{T}$ cells undergo either attraction or repulsion in the in vitro assays. For this reason, it cannot be

excluded that different subsets of cells, with intrinsic differences that favor one response or the other, exhibit chemorepulsion or chemoattraction. The increasing effort that is being directed at determining how a cell can sense different concentrations of ligand across its length to make a chemotactic response should help in developing an understanding of how a chemokine-mediated repulsive response might occur.

\section{Chemorepulsion in vivo?}

Having found suggestive in vitro evidence for lymphocyte chemorepulsion/fugetaxis, the Scadden group (2) sought to identify an in vivo example of the process. Revisiting a decade-old observation - that cell emigration from the thymus is inhibited by the ADP-ribosylating subunit of PTX (6) Poznansky and coworkers (2) examined a variety of thymic culture systems and cell types and showed that mature cells exit thymic fragments or organoids in a PTX-inhibitable manner. Similarly, Lee et al. (7) recently reported that the PTX-treated cells accumulate inside thymic vascular channels, structures that may be associated with $T$ cell exit. In support of a role for chemorepulsion in thymocyte emigration, Poznansky et al. found that if thymic stroma-conditioned medium was placed in the lower Transwell chamber, cells no longer migrated as efficiently away from the thymic organoids. The thymus is an established source of SDF1/CXCL12, and antibodies to the SDF1/CXCL12 receptor, CXCR4, could partially inhibit cell emigration from the thymic cultures. Switching to $\mathrm{CD}^{+} \mathrm{T}$ cells as the indicator population, Poznansky et al. showed that thymic stroma-conditioned medium contains another, possibly chemorepulsive, activity. In this case, however, it was not excluded that this activity was purely chemokinetic. Moreover, since several of the systems that have been used to assess this effect are quite complex, alternatives to true chemorepulsion have not always been ruled out. In particular, if thymocyte motility increases as these cells mature, the effect would mimic that of chemorepulsion in some experiments. Interestingly, Lee and colleagues recently reported that positive selec- 
tion in fetal thymic organ culture is indeed associated with increased motility of the selected cells (7).

Analysis of the SDF1/CXCL12CXCR4 contribution to thymocyte emigration in vivo has been difficult, as deficiency for either gene causes perinatal lethality. However, studies of lethally irradiated mice reconstituted with CXCR4-deficient fetal liver or lymphocyte-deficient mice grafted with CXCR4-deficient fetal thymi appear to indicate that CXCR4 is not limiting in thymocyte emigration (8, $9)$. Further studies are needed, for example, in mice selectively lacking CXCR 4 in T cells, before we can fully understand the role of this receptor in thymocyte development.

In general, it is difficult to judge from these in vivo studies whether direct chemorepulsion is involved in thymocyte motility. In this regard, it is interesting to consider another recent report, indicating that ELC/CCL19 and its receptor CCR7 participate in thymocyte exit, again possibly through a chemorepulsive mechanism. Addition of ELC/CCL19 to the medium of fetal thymic organ cultures strongly augments thymocyte emigration (10). Mice lacking CCR7 or mice treated with an ELC/CCL19-neutralizing polyclonal antibody have slightly increased total numbers of thymocytes and reduced numbers of $\mathrm{T}$ cells within the spleen, although, surprisingly, they do not have increased proportions of mature singlepositive CD4 or CD8 T cells in the thymus, in contrast to what is observed in PTX transgenic mice (6). In addition, adult CCR7-deficient mice accumulate approximately normal numbers of peripheral $\mathrm{T}$ cells, making it clear that the CCR7 pathway cannot provide the whole explanation for thymocyte emigration. Interestingly, ELC/CCL19 is expressed at higher levels in medullary epithelial cells than in the adjacent endothelial structures that are believed to be associated with exit (10), consistent with the idea that this factor helps drive thymocyte emigration by a chemorepulsive mechanism.

Overall, while there is still much to be learned, the new studies are significant in that they develop systems for studying thymocyte exit and they provide hints as to the pathways that are likely to be involved. The findings on chemorepulsion are tantalizing and should certainly prompt further investigation of this process, particularly in systems where the chemokine concentration gradient can be precisely quantified and controlled. It will also be interesting to see whether molecules established to have repellent functions in the nervous system, such as members of the ephrin and semaphorin families (see Perspective by Goshima et al. [ref. 1]), participate in chemorepulsion of lymphoid cells and whether, like neurons, lymphoid cells can undergo intrinsic changes that switch their response to a ligand from attractive to repulsive.

1. Goshima, Y., Ito, T., Sasaki, Y., and Nakamura, F. 2002. Semaphorins as signals for cell repulsion and invasion. J. Clin. Invest. 109:993-998. DOI:10.1172/JCI200215467.

2. Poznansky, M.C., et al. 2002. Thymocyte emigration is mediated by active movement away from stroma-derived factors. J. Clin. Invest. 109:1101-1110. DOI:10.1172/JCI200213853.

3. Poznansky, M.C., et al. 2000. Active movement of $\mathrm{T}$ cells away from a chemokine. Nat. Med. 6:543-548.

4. Zlatopolskiy, A., and Laurence, J. 2001. 'Reverse gear' cellular movement mediated by chemokines. Immunol. Cell Biol. 79:340-344.

5. Giger, R.J., and Kolodkin, A.L. 2001. Silencing the siren: guidance cue hierarchies at the CNS midline. Cell. 105:1-4.

6. Chaffin, K.E., and Perlmutter, R.M. 1991. A pertussis toxin sensitive process controls thymocyte emigration. Eur. J. Immunol. 21:2565-2573.

7. Lee, C.K., et al. 2001. Thymic emigrants isolated by a new method possess unique phenotypic and functional properties. Blood. 97:1360-1369.

8. Zou, Y.R., Kottmann, A.H., Kuroda, M., Taniuchi, I., and Littman, D.R. 1998. Function of the chemokine receptor CXCR4 in haematopoiesis and in cerebellar development. Nature. 393:595-599.

9. Ma, Q., Jones, D., and Springer, T.A. 1999. The chemokine receptor CXCR4 is required for the retention of $\mathrm{B}$ lineage and granulocytic precursors within the bone marrow microenvironment. Immunity. 10:463-471.

10. Ueno, T., et al. 2002. Role for CCR7 ligands in the emigration of newly generated $\mathrm{T}$ lymphocytes from the neonatal thymus. Immunity. 16:205-218. 